\title{
La prise en compte des partenaires dans l'œuvre de santé : innovations et experimentations
}

Christian Moreau

\section{(2) OpenEdition}

1 Journals

Édition électronique

URL : http://journals.openedition.org/communicationorganisation/2990

DOI : 10.4000/communicationorganisation.2990

ISSN : $1775-3546$

Éditeur

Presses universitaires de Bordeaux

Édition imprimée

Date de publication : 1 mai 1994

ISSN : 1168-5549

Référence électronique

Christian Moreau, « La prise en compte des partenaires dans l'œuvre de santé : innovations et experimentations », Communication et organisation [En ligne], HS N¹ | 1994, mis en ligne le 27 mars 2012, consulté le 24 avril 2019. URL : http://journals.openedition.org/

communicationorganisation/2990 ; DOI : 10.4000/communicationorganisation.2990

Ce document a été généré automatiquement le 24 avril 2019

(c) Presses universitaires de Bordeaux 


\title{
La prise en compte des partenaires dans l'œuvre de santé : innovations et experimentations
}

\author{
Christian Moreau
}

\section{NOTE DE L'ÉDITEUR}

Présentation et animation de la table ronde Christian Moreau

1 Je vais vous présenter très brièvement les intervenants qui seront à cette tribune.

2 Pour commencer, Sylvie GOLDBERG, Responsable de la Direction de la communication de la Direction des hôpitaux, au ministère des affaires sociales, de la santé et de la ville, qui présentera tout à l'heure une intervention sur les réseaux de chargés de communication hospitalière. À côté d'elle, Nadège MARECHAL, qui est Sociologue à l'hôpital Max FORESTIER de Nanterre; elle travaille actuellement au Centre d'Accueil et de Soin Hospitalier, dont les initiales sont CASH, connotation marketing si l'on peut dire pour un établissement public, et elle va nous faire part d'une expérience qu'elle a menée dans un établissement où elle travaillait préalablement, le CHU de Rouen, sur les relations partenaires-hôpital à propos des malades du sida. À côté de moi, Patricia FRAYSSE, Chargée de communication au Centre Hospitalier d'Angoulême, qui nous présentera une démarche liée à l'opération Pièces jaunes-Soleil, et aussi des actions de prévention menées par l'hôpital en collaboration avec d'autres partenaires. Jean-Claude FINES, qui est Président de l'association d'entraide généraliste de Bordeaux. Marie-Paule BEAUFILS, Responsable de communication à l'hôpital Bichat Claude-Bernard, Assistance Publique des Hôpitaux de Paris, qui a menée une opération d'élaboration de fiches pour mieux faire connaître l'hôpital Bichat auprès de ses partenaires que sont les médecins de ville, en partenariat avec un laboratoire pharmaceutique. 
3 Je vais donc laisser la parole dans un premier temps à Nadège MARECHAL, qui va nous parler des relations entre les partenaires de l'hôpital à propos des malades du sida.

\section{Les relations entre les partenaires de l'hôpital à propos des sidéens (Nadège MARECHAL Sociologue, Hôpital Max FOURESTIER de Nanterre)}

4 En ouvrant cette table ronde consacrée à la prise en compte des partenaires de l'hôpital dans l'œuvre œuvre de santé, je me vois dans l'obligation de commencer mon exposé par un bref rappel de l'organisation hospitalière, avant d'illustrer mes propos à partir de deux études que j'ai mené dans le service de Maladies Infectieuses du CHU de ROUEN sur la prise en charge des malades séropositifs et sidéens.

5 Lieu historique d'accueil, l'hôpital public est progressivement devenu une structure d'hébergement et de soins, avant de s'imposer comme un pôle de compétence technique autour duquel s'est organisé l'ensemble du système sanitaire.

6 Outre les bouleversements majeurs que le monde hospitalier connait aujourd'hui, l'étude du fonctionnement de l'hôpital révèle combien le malade est instrumentalisé, contraint à la passivité, sous le poids des impératifs organisationnels.

7 L'individu est souvent réduit à sa pathologie autour de laquelle s'élabore l'organisation hospitalière.

8 L'organisation du temps et de l'espace à l'hôpital obéit à des règles qui répondent pour beaucoup en priorité aux besoins des services et de leurs personnels (horaires des consultations, des laboratoires).

9 L'irruption dans le champ hospitalier de nouvelles catégories d'usagers bousculent les habitudes hospitalières, l'exemple des sidéens en est l'illustration la plus éclatante.

10 Afin de pallier les problèmes d'affluence rencontrés par les services de Maladies infectieuses dans la prise en charge des patients séropositifs et sidéens, la création de réseaux de ville composés de médecins généralistes a été institué par une circulaire du 4 juin 1991.

11 Le réseau est une liste de médecins de ville à la disposition des malades garantissant à ces derniers une collaboration étroite avec les praticiens hospitaliers.

12 L'objectif des deux études était de mettre en exergue les réactions et attentes des médecins et des malades à l'égard de ce réseau mais aussi d'évaluer leur degré d'adhésion à une telle entreprise.

13 Ces deux études basées à partir de 40 entretiens qualitatifs se sont déroulées sur la région rouennaise en décembre 90 pour les médecins et juillet 91 pour les malades.

\section{Les médecins libéraux}

L'étude a démontré un certain comportement " attentiste »; il ne faut pas comprendre ce terme comme étant un manque d'intérêt de la part des médecins pour cette nouvelle pathologie mais plutôt comme une traduction de la manière dont s'exerce la médecine générale dans les cabinets de ville. 
15 Tous les médecins confirment qu'il est de leur devoir de prendre en charge des malades et d'assurer le suivi des maladies intercurrentes (qui survient au cours d'une autre), par contre ils rejettent la possibilité d'assurer, compte tenu des structures actuelles, cette maladie lors de complications majeures.

16 Cette limite traduit bien l'exigence des praticiens à conserver leur rôle de médecins généralistes. En revanche, ils revendiquent le droit de ne pas vouloir prendre en charge des malades toxicomanes devant les difficultés qu'ils rencontrent dans leur suivi.

17 Ils adoptent alors une seule démarche, suivre leur propre patient ; ils souhaiteraient être informé de leur rôle lors du développement de la maladie.

\section{Les patients}

18 L'annonce de la séropositivité est le moment précis où se définit l'attitude du patient à l'égard du généraliste: soit elle se concrétise rapidement par une relation privilégiée ; soit elle est difficile et le malade a donc recours aux médecins hospitaliers comme le lui conseille très fortement son médecin.

19 Pour pouvoir être suivi par un médecin traitant dans le cadre de cette maladie, il faut impérativement avoir confiance en son médecin traitant, non seulement au point de vue médical mais aussi sur le respect de la confidentialité.

20 Il faut prendre en compte le fait que ce sont des patients jeunes ayant rarement un médecin traitant.

21 Les malades pensent que leurs médecins sont très peu, voire mal informés sur cette maladie et ressentent même quelques réticences des médecins à l'égard de leur cas médical. Le témoignage de certains patients dévoile bien souvent des maladresses. Se sentant rejetés par la médecine de ville, certains patients s'avouent totalement dépendants du médecin hospitalier.

22 L'hôpital devient le recours irremplaçable car la relation est très forte avec l'hospitalier d'autant que dans la majorité des cas le praticien hospitalier est le premier intervenant du corps médical à prendre en charge la maladie; de plus les malades viennent régulièrement à l'hôpital pour les bilans ou traitements.

23 Toutefois malgré un suivi régulier à l'hôpital, quelques malades assignent à leur médecin de ville un rôle relativement limité (renouvellement de l'arrêt de travail ou prescription de médicaments).

24 Dans ce cas, le patient assure bien des fois le rôle d'intermédiaire entre l'hôpital et le médecin traitant ; il informe son médecin des résultats des bilans, des différents examens qui lui ont été faits et du traitement que le praticien hospitalier lui a prescrit.

\section{Conclusion}

Comme nous l'avons vu précédemment, la prise en charge d'un suivi médical et social d'un séropositif implique certaines règles de pratiques et de conduites tant du côté des médecins libéraux et hospitaliers que du côté des malades.

Dès l'annonce de la séropositivité, le malade plonge très rapidement dans un milieu où son rapport avec le médical est omniprésent. 

tout à l'heure, l'hospitalo-centrisme a vécu, mais il n'est pas mort, d'où la difficulté que l'on peut rencontrer concrètement à établir un réseau ville-hôpital. l'hôpital BICHAT-CLAUDE BERNARD, Assistance Publique Hôpitaux de Paris, qui a élaboré un classeur de fiches d'information destiné à la fois aux médecins de ville, pharmaciens d'officine, biologistes, chirurgiens dentistes, infirmières libérales, assistantes sociales, bref à plusieurs partenaires de l'hôpital.

Elle va donc vous présenter le résultat de cette opération qui, elle vous le dira elle-même, est le fruit d'un partenariat à trois : hôpital, médecins de ville et aussi un laboratoire prédestiné de par son nom à travailler avec l'hôpital Bichat, puisqu'il s'agit du laboratoire BEECHAM. 

Peut-être que certaines personnes présentes dans la salle ne connaissent pas cet établissement. Mais sur la France, pour les spécialistes, et même sur un plan mondial, il est extrêmement connu pour ses pathologies. Jean-François TOME, lors de son intervention, a précisé que des audits avaient été réalisés à l'Assistance Publique, sur la relation médecine de ville-médecine hospitalière. Les résultats de ces analyses ont été très mauvais. Lorsque je suis arrivée au service communication de l'hôpital BICHAT, j'avais toujours en tête l'idée d'améliorer nos relations avec tous les partenaires, associations comprises. Je me suis basée sur un audit qui avait été réalisé à l'hôpital ROTHSCHILD et à l'hôpital LARIBOISIERE, parce que je ne voulais pas refaire une autre analyse à BICHAT. Il s'avère que tout ce que l'on pouvait reprocher à ROTHSCHILD, était semblable à ce que l'on pouvait reprocher à BICHAT. A savoir que les médecins généralistes étaient très mal accueillis, perçus comme des sousmédecins, que les informations ne leur étaient pas données, le suivi de leurs malades et les comptes-rendus ne leur étaient pas transmis, quelquefois on leur "court-circuitait " leurs patients, et on ne les informait pas. En fait, il y avait tout un ensemble de choses qu'il fallait absolument améliorer. Lorsque la nouvelle commission médicale consultative est arrivée, il s'est créé à l'intérieur une commission médecine de ville. Un des chefs de service est venu me voir, et nous avons décidé de réaliser quelque chose pour améliorer ces relations. Nous avons essayé de réfléchir à un projet, et entre-temps, des praticiens hospitaliers, Professeurs des universités (P.U.P.H), ont rencontré des amicales de médecins de ville. Les remarques qui ont été faites, ont été absolument les mêmes que celles que faisait ressortir l'audit. Alors, nous avons organisé des réunions auxquelles ont participé des administratifs, des médecins P.U.P.H, des praticiens hospitaliers. Un accord a été trouvé, nous avons décidé de monter un projet. Nous avons bâti une trame, une fiche-type, adressée à tous les chefs de service en leur demandant de la remplir, d'apporter les modifications, les annotations qu'ils souhaitaient. Une question se posait parallèlement : qui allait financer ce projet? Nous avions des rapports assez privilégiés avec l'Institut BEECHAM ouvert à toute éventuelle coopération médicale. Le moment était donc venu de les solliciter. J'ai demandé à mon directeur de présenter par écrit le projet au directeur général de l'Institut BEECHAM des laboratoires BEECHAM. Ce dernier a adhéré au projet, il a accepté d'assurer le financement. Nous avons alors lancé le travail de l'imprimeur et le projet a abouti.

Cela a demandé un certain temps bien sûr, car BICHAT est un établissement très important. 11 nous a fallu aller à la "pêche » aux renseignements. Le document a été édité, BEECHAM a souhaité monter une manifestation conviviale autour de ce partenariat. Nous avons donc organisé un cocktail, auquel ont été invités des médecins généralistes, des associations, des amicales, le conseil de l'ordre, le doyen de la faculté, et les chefs de service de Bichat. Nous leur avons remis officiellement ce jour-là ce dossier « Médecine de ville », et la diffusion a été assurée par le laboratoire, qui donnait, par l'intermédiaire des délégués médicaux, le dossier aux médecins de ville.

Je dois rajouter que les médecins de ville sont nos partenaires. C'est pourquoi j'appelle cela une trilogie, car je considère que nous devons faire un effort pour informer les médecins des ville et surtout les accueillir. Ce sont eux qui nous amènent les malades. Si nous n'avons pas un minimum d'égard pour les recevoir, ils peuvent toujours aller voir ailleurs, même si BICHAT est très connu. De plus, nous pouvons être en compétition 
même à l'intérieur de nos hôpitaux Assistance Publique. D'après une des idées de notre direction générale, c'est à celui qui sera le plus efficace, le plus accueillant, et je crois que c'est là que se fera la différence.

\section{Questions}

\section{MOREAU : Quel est son coût?}

Marie-Paule BEAUFILS : Je peux vous dire ce qu'il a coûté au laboratoire : $230000 \mathrm{~F} 11$ a été tiré à 3000 exemplaires. Nous n'avons ciblé que la médecine générale, parce que nous savons que les médecins spécialistes ont tous leurs correspondants à l'hôpital, donc ils n'ont pas besoin d'être informés. Par contre, les médecins généralistes ne savent pas lorsqu'ils ont un problème avec leurs patients, à qui s'adresser spécifiquement à l'hôpital.

C'est un document très informatif, qui ne dit pas que nous sommes les meilleurs, les plus beaux, les plus forts. C'est juste une information, un correspondant, une surveillante, un numéro de téléphone.

50 J'ai quand même pensé à Assistance Publique Hôpitaux de Paris, car j'ai mentionné le service 3614 code APHP, qui peut fournir des informations sur toute l'Assistance Publique Hôpitaux de Paris.

51 François BADENES : Au-delà des phases d'élaboration, des aspects financiers, de l'objectif, $\mathrm{y}$ a t-il derrière tout cela un suivi, puisque cela demande, je l'imagine, des remises à jour régulières?

Marie Paule BEAUFILS : Il n'y a pas encore de remise à jour, puisque ce document a été édité en septembre de l'année dernière. La remise à jour se fera, je pense, à la fin de cette année.

Quant aux retombées, celles-ci sont données au laboratoire. Ce dernier nous informe que les médecins généralistes sont ravis d'avoir un document comme celui-ci, et qu'ils apprécieraient que tous les établissements fassent de même, car cela pourrait être un moyen d'information sûre. Je voulais dire également que nous avons fait un plan, un répertoire afin de présenter notre groupe hospitalier en quelques chiffres et mots très courts.

M.MOREAU : Nous allons passer la parole à Patricia FRAYSSE, chargée de communication au Centre Hospitalier d'ANCOULEME, auparavant au même poste au Centre Hospitalier de LAVAL, elle va nous parler de ses expériences de LAVAL et d'ANCOULEME.

Patricia FRAYSSE, Chargée de communication, Hôpital Général d'ANCOULEME

Trois objectifs sont poursuivis, lorsque l'on recherche des partenaires :

\section{1- Recherche de partenaires dans les actions de prévention :}

a) pour donner de la cohérence à un message et éviter la redondance des informations :

Ainsi, il a été mené une action au Centre Hospitalier d'Angoulême pour la Journée Mondiale sur le SIDA. Compte tenu du thème, il était nécessaire de cibler les collèges et les lycées. De très nombreuses associations avaient préparé un programme : la P.M.I., le Centre Hospitalier Spécialisé, la Délégation à l'Information contre la drogue, des associations plus modestes. Afin que l'information soit coordonnée, le service de médecine scolaire de l'Inspection Académique a été contacté. On s'est aperçu que chaque 
programme avait été transmis à un même lycée, situé en centre-ville. Les lycées ruraux n'avaient rien reçu.

Retraités. En effet, des conférences sont organisées et pour assurer l'information, on peut utiliser le réseau de l'office. Ainsi, on touche des publics qui ne font pas partie des institutions. De même, le cycle de conférences peut être rendu divertissant dans la mesure où il existe de nombreux clubs de chants ou de danses folkloriques qui peuvent organiser des spectacles de courte durée entre les conférences.

La réunion s'étant faite tardivement, la coordination n'a pas pu être faite. Mais, un seul programme a été fait par la suite pour être diffusé le plus largement possible. institutions s'effacent devant les messa faire de la publicité en récupérant les actions.

Sur un plus long terme, il a été convenu, et pour éviter les écueils, que des réunions régulières puissent avoir lieu pour coordonner les compétences de chacun.

b) faire partager les compétences et aborder, compte tenu de l'expérience des partenaires, les diverses facettes des thèmes retenus pour la campagne de prévention.

Certaines actions doivent allier compétences techniques et compétences pédagogiques.

Actions sur le petit-déjeuner :

$\mathrm{Au}$ moment de la rentrée des classes, une action a été menée sur ce thème en invitant les écoles du quartier. L'hôpital disposait de diététiciennes, compétence technique pouvant apporter des réponses sur l'équilibre alimentaire et présenter des plateaux équilibrés. Mais, il faut aussi mettre en place une exposition adaptée au public reçu, des vidéos, diverses animations. C'est pourquoi, une action de partenariat s'est organisée avec le Centre d'Examen de Santé de la CPAM et la Mutualité Française. Ainsi, les enfants ont reçu à la fois un message technique et pédagogique.

Par ailleurs, cette collaboration continue puisqu'une diététicienne de l'hôpital collabore aux actions menées dans les écoles.

Des actions sur le SIDA, le tabac ont été menées également avec ces mêmes partenaires et aussi le Comité d'Education pour la Santé et l'École d'Infirmières.

Dans le cadre de Journées Santé organisées par la Caisse Primaire d'Assurance Maladie, l'ensemble des hôpitaux du Département de la Mayenne se sont regroupés sur un stand commun intitulé "La prévention et l'hygiène de vie ». Avec le réseau d'adresses de chaque structure, des stands dynamiques ont été organisés : flipper santé, théâtre-forum, logiciels...

c) toucher des publics variés

Au moment de la Semaine des Personnes Agées, il est utile de travailler avec les Offices de Une collaboration intéressante s'était aussi établie au Centre Hospitalier de Laval lors d'une campagne sur le don du sang.

Une action avait été menée avec la section BTS Force de Vente qui avait réalisée une affiche pour sensibiliser leurs jeunes collègues. La collecte de sang réalisée auprès des jeunes de ce lycée avait été très importante.

d) se faire mieux connaître

Lors d'une action Sport et Santé organisée à Angoulême en juin 1993, de nombreux lycéens ont découvert un service de médecine sportive peu connu jusqu'alors. 
75 Lors d'une campagne antitabac menée à Laval, nous avons monté des actions de prévention (logiciel ; tests d'odeurs ; goûtez à l'eau...) avec la collaboration du Comité d'Education pour la Santé, les élèves-infirmières de la Croix Rouge, les médecins hospitaliers. Nous avons lancé une consultation d'aide au sevrage tabagique, à ce moment-là.

76 Par ailleurs, nous avons commencé sur ce thème, une action de partenariat avec le Centre Socio-culturel du quartier.

77 e) rassembler les outils pédagogiques existants, sans perdre du temps à essayer de chercher ou de réinventer des produits existants proches de notre lieu d'action.

78 Echanger, fédérer, coordonner et communiquer, tels sont les mots à retenir dans une recherche de partenaires dans les actions préventives.

79 L'hôpital a besoin de partenaires pour aussi mettre en place un projet local. Ici, la recherche a pour but de faire financer par des fonds extra-hospitaliers des projets d'équipement ou d'animation qui n'auraient pas vu le jour.

\section{2 - Recherche de fonds}

80 a) Dans le but d'améliorer l'accueil des enfants hospitalisés, la Fondation de France a lancé l'OPJ (Opération Pièces Jaunes). Cette opération consiste à récolter le plus de fonds possibles en pièces jaunes par l'intermédiaire de la Poste. Le CHA (Centre Hospitalier d'Angoulême) avait pour les services de pédiatrie, un projet d'aménagement d'une aire de jeux à l'extérieur. En 1993, une campagne d'information a été menée sur ce projet. Un dépliant spécifique sur ce projet réalisé en collaboration avec la DDJSS (Direction Départementale de la Jeunesse et des sports) a été édité (maquette jeunesse et sports ; imprimerie : CHA). Les réseaux de diffusion ont été : par l'intermédiaire de Jeunesse et Sports, les Maisons des Jeunes et de la Culture; par la Mairie, l'ensemble des services municipaux; le journal interne de la Poste; la Caisse d'Allocations Familiales. Une subvention de 115000 francs a été obtenu.

81 En 1994, nous avons relancé cette opération dans le but d'obtenir un des trois premiers prix de Communication Hospitalière. Le CHA a obtenu le troisième prix, soit une subvention de 100000 francs. Les réseaux de diffusion ont été nombreux et variés : les partenaires institutionnels se sont mobilisés: la chargée de communication de la Chambre de Commerce et d'Industrie a adressée une lettre de motivation aux entreprises charentaises accompagné du matériel pédagogique affiches et tirelires.

La Caisse Primaire d'Assurance Maladie a fait une information interne et mis dans chaque guichet d'accueil une tirelire et une affiche.

83 La Mairie a écrit une lettre aux 60 écoles publiques et privées d'Angoulême, a mis des tirelires dans les services municipaux.

84 La Société de Bus des Transports Angoumoisins a installé des affiches dans tous les bus pour annoncer l'Opération.

85 L'Opération Pièces Jaunes, grâce toujours aux affiches et tirelires a été connue lors du Festival de la Bande Dessinée ; quelques stands ont accepté d'avoir affiches et tirelires. De même, lors du Salon du Jouet qui s'est déroulé récemment «Ludoland ». Grâce à la Poste, une soirée a pu être organisé lors d'une manifestation qu'elle parrainait nommée Festifoot qui réunit tous les clubs de football du Poitou-Charentes. Grâce à une 
collaboration que nous avons réussi à mener avec l'École Nationale de Musique d'Angoulême, les professeurs de musique ont distribué une tirelire à chaque enfant. De même le Conservatoire a parrainé un concert au profit de l'hôpital et notamment du Centre de Dépistage du SIDA. Des recettes lui ont été versées. Grâce à des financements de type privés, des actions ont pu être lancées.

\section{3 - L'hôpital aidant} de fonds. Ainsi, le Centre Hospitalier de Laval a aidé l'Association de lutte contre la Mucoviscidose. Un pin's avait été réalisé avec le logo de chaque partenaire et vendu à l'accueil. Une information avait été faite dans chaque service pour assurer la diffusion et expliquer le fonctionnement des Tirades de l'Espoir, course organisée en septembre. Un partenariat avait été aussi mené avec une mutuelle. L'hôpital a pu remettre à l'Association un chèque de 60000 francs.

\section{Conclusion}

Comme ces expériences ont pu le montrer, la recherche des partenaires selon le type d'opérations poursuit des buts différents. L'hôpital ne peut plus travailler seul, il doit s'intégrer dans la cité. 11 doit aussi constituer un pôle d'attraction. Souvent une des premières entreprises, il doit être le moteur de l'action et donner une image positive et dynamique de l'institution. Cela participe à l'information du public et plus largement à son accueil. Il se fait connaitre et lorsque l'habitant devient usager, il ne rentre plus dans un monde secret et mystérieux.

M. MOREAU : Sylvie COLDBERC va nous présenter le réseau de chargés de communication hospitalière qu'elle anime, elle est aussi responsable, fondatrice du journal « Informations hospitalières ", qui joue un rôle important dans la communication hospitalière et dont je vous présente ce numéro hors-série, un "guide de la communication hospitalière ", préfacé par le directeur général du CHU de Bordeaux.

\section{Sylvie COLDBERC, Direction de la Communication,, Direction des hôpitaux., Ministère des} affaires sociales nécessité et la manière de la développer tant pour le bien-être du malade que pour améliorer l'organisation et les relations de travail, tel a été le souci de la Direction des Hôpitaux dans la démarche qu'elle a entreprise en 1992.

Quel a été le rôle du Ministère dans cette démarche?

Un soutien et un encouragement à la création de partenariats :

- entre hôpitaux,

- entre hôpitaux à l'intérieur d'une même région,

- entre les hôpitaux et d'autres instances.

En réunissant pour la première fois en septembre 1992, l'ensemble des responsables de la communication des hôpitaux, la Direction des Hôpitaux a voulu leur permettre de se connaître et de réfléchir ensemble à une culture de communication commune au monde hospitalier et affirmer ainsi cette spécificité. 
97 La première réunion nationale a été l'occasion de créer un réseau dont les actions devraient permettre de développer progressivement les moyens appropriés au secteur qui nous préoccupe en ayant bien à l'esprit sa dimension humaine.

Ces actions se sont concrétisées par la mise en place de groupes de travail sur des thèmes qui nous ont paru important de traiter pour la première année et qui ont fait l'objet d'une publication: «Le Guide pratique de la communication hospitalière » numéro hors série de la revue Informations Hospitalières paru en octobre 1993 dont la préface est signée de Pierre LE MAUFF, Directeur Général du Centre Hospitalier Universitaire de Bordeaux, qui nous a encouragés dans notre initiative.

D'autres thèmes sont traités en 1994 et feront l'objet d'une nouvelle publication lors de la troisième réunion nationale qui aura lieu à l'automne 1994. la communication à l'hôpital. D'abord, à la communication interne afin de développer une meilleure participation. Toute recherche d'adhésion autour d'un projet suppose une bonne information des partenaires. À la communication externe ensuite pour assurer plus de transparence entre l'hôpital et les usagers ainsi qu'avec les divers acteurs de la santé pour une meilleure prise en charge du malade lorsqu'il quitte l'hôpital.

Si la mission de soin de l'hôpital est une finalité première, l'information qui contribue directement à sa qualité, revêt une importance que nous ne saurions négliger.

Nous avons encouragé et soutenu différents types de partenariats :

1 - Partenariats régionaux :

105 Je voudrais citer en exemple l'expérience de la région Nord-Pas-de-Calais dont les responsables de la communication se sont associés en pôle régional. L'AP-HP en a fait autant avec l'ensemble des chargés de communication de ses 50 établissements.

Des expériences de ce type méritent d'être encouragées pour qu'elles servent de relais professionnel.

1072 - Partenariat avec d'autres entreprises n'appartenant pas au monde hospitalier

108 Depuis quelques mois, nous avons franchi une nouvelle étape, celle de l'ouverture à d'autres secteurs. Si la communication ne peut s'appliquer à l'hôpital comme elle peut s'appliquer à d'autres secteurs de l'activité économique, elle peut avoir des points communs avec certains d'entre eux.

La confrontation et le partage des expériences sont toujours source d'enrichissement :

110 Par exemple les rencontres et le rapprochement avec des journalistes et avec l'École de journalisme de Lille dans notre souci partagé d'information du malade.

111 Une réflexion sur la déontologie régissant les métiers de l'information et ceux de la santé commence à être menée au sein d'un groupe de travail.

112 Plus surprenant peut paraitre le lien que nous avons tissé avec « Aéroports de Paris »

113 Les aéroports comme les hôpitaux sont des lieux où la charge de stress est très importante, d'où l'intérêt de ce rapprochement que je pourrai développer si vous le souhaitez lors de la discussion.

114 A travers le travail qui a été fait depuis près de deux ans, les différents groupes de travail ont cherché à définir comment pouvait se concevoir la fonction de communication dans 
le contexte hospitalier, comment elle pouvait s'inscrire dans la stratégie de l'hôpital, mais également définir sa place dans l'équipe hospitalière.

La Direction des hôpitaux a ainsi donné le coup d'envoi d'une réflexion par les intéressés eux-mêmes pour en faire bénéficier l'ensemble des acteurs de la communication à l'hôpital.

116 M.MOREAU : Je vais maintenant passer la parole à Jean-Claude FINES, qui va vous présenter le type d'intervention qu'il réalise avec son association à l'hôpital, des interventions culturelles avec des artistes, ou des interventions très variées.

117 Jean-Claude FINES, Association d'entraide générale-Dépannage

118 Dépannage est une association loi 1901 créée il y a un an à Bordeaux par la volonté de trois amis d'aider les enfants en difficulté.

L'idée est simple: distraire et amuser les enfants en situation affective ou physique anormale, qu'ils soient hospitalisés ou placés dans des centres spécialisés.

Que faisons nous?

Dépannage s'occupe de trouver les fonds nécessaires au financement de distractions ou de matériels (jeux, jouets, Hi-Fi, T.V...) pouvant aider ces enfants en situation difficile. Nous avons essentiellement mis en place un service régulier de "Journées distractions » sur le C.H.R. de Bordeaux. 11 s'agit de mini-spectacles donnés chambre par chambre, de façon à pouvoir distraire sans distinction les enfants valides ou non. Ces spectacles prennent la forme de représentations diverses : clowns, ventriloques, marionnettistes, tours de magie ou encore initiations aux instruments de musique. Ils sont donnés par des personnes choisies à la fois pour leur expérience professionnelle antérieure avec les métiers de l'enfance (instituteurs, éducateurs spécialisés..) mais également pour leurs compétences artistiques. L'expérience acquise tout au long de cette année en fait des spécialistes qui savent instaurer un lien particulier avec l'enfant.

À l'heure actuelle, environ cinquante spectacles ont été organisés sur l'ensemble du CHR. Ces actions se font sous l'égide du personnel hospitalier (surveillante, surveillante-chef..) à qui il est continuellement demandé de porter une appréciation et de renouveler l'autorisation d'intervenir.

De multiples réactions positives ont été recueillies notamment auprès du personnel soignant. Cela nous motive pour aller plus loin dans la recherche de nouvelles activités ludiques et dans la volonté de multiplier la fréquence des interventions ainsi que le nombre des établissement bénéficiaires.

124 Le financement de Dépannage est basé sur la collecte de dons de particuliers ou d'entreprises sensibilisées à l'action que nous menons.

\section{Questions}

Marie-Paule BEAUFILS: 11 faut énormément tenir compte des associations dans les établissements de santé. Parce ce que ce sont des partenaires qui apportent beaucoup à l'hôpital, ils financent des choses que l'hôpital ne peut pas financer. D'abord, ils apportent un bien être pour le malade, sur un plan relationnel, mais en plus, quand on les connait bien, que l'on a de bonnes relations avec eux, ils vous proposent des idées auxquelles vous n'auriez pas pensé, et ils financent des projets que l'hôpital ne financera jamais. Par exemple, l'équipement d'un bureau, l'achat de matériel... 
126 Nous, nous avons une association, «le Mouvement pour l'amélioration de l'environnement hospitalier ", qui nous offre, pour une chambre de malade complètement dépendant, la TV qui fonctionne à la voix, l'électricité... ce que l'hôpital n'aurait jamais pu financer. il est vrai que ce sont des partenaires dont il faut absolument tenir compte. De toute façon, il y a des associations qui sont beaucoup plus impliquées dans la pathologie, notamment les associations d'aide qui, si vous ne les prenez pas en compte, savent rappeler leur présence. Ce sont des gens qui font des choses vraiment remarquables.

François BADENES : Tout d'abord, je voulais dire un grand bravo à M.FINES. C'est vrai que nous avons besoin des associations. Nous travaillons souvent avec elles, ce sont des partenaires privilégiés. Mais, je voulais dire également que la structuration des réseaux de chargés de communication, dont parlait tout à l'heure Mme GOLDBERG, n'existe pas vraiment concrètement aujourd'hui. En effet, on rencontre des profils très différents, des formations très différentes. Cela est en train de se mettre en place progressivement, et je dirais que cela dépend à chaque fois de la volonté du directeur de communiquer, de mettre des moyens dans la communication de son hôpital. De la même façon, malgré les incantations à l'amélioration de l'accueil dans les hôpitaux, on sait tous hélas, que l'on a tendance à mettre des « bras cassés » aux pôles d'accueil, au standard, et à d'autres postes clés de l'hôpital. Tout cela en raison des contraintes budgétaires, est en train de progresser, mais jusqu'à maintenant, il n'y avait pas vraiment eu de réflexion globale sur ces points. Les associations, les laboratoires nous en avons besoin ; mais c'est quelque part un peu malsain, de devoir compter sur eux parce que l'on se lie à eux. Nous sommes obligés de créer des associations pour avoir une souplesse de financement, parce que lorsque nous voulons monter une opération et que nous annonçons aux partenaires que nous allons mettre 6 mois avant de les payer, cela ne se fait pas tout seul. Nous créons donc des associations pour faire rentrer de l'argent par des laboratoires, auxquels nous sommes bien obligés ensuite de donner quelque chose. C'est dans cette nébuleuse, parfois un peu difficile à gérer, qu'il y aurait besoin de clarification pour avoir les moyens de nos ambitions en terme de communication.

Thérèse PSUIK : Pouvez-vous nous préciser les relations que vous avez avec le personnel soignant?

Jean-Claude FINES : Cela dépend des endroits. Elles sont relativement bonnes, en ce sens où nous sommes d'emblée placés. Nous avons été demandeurs au départ puisque nous sommes venus proposer un service. Ensuite nous avons laissé venir un petit peu. À savoir, vous avez besoin de quelque chose, vous savez que nous sommes là. Je ne rencontre pas tous les matins les directeurs de l'hôpital de Bordeaux, bien sûr, ni les surveillantes... À notre niveau, et lorsqu'il y a besoin, ils savent nous trouver (directeurs d'hôpitaux, responsables, infirmières en chef...). Je pense que c'est simple. Nous ne cherchons pas à révolutionner le monde hospitalier.

Thérèse PSUIK : Ma question était en fait plus précise. Selon moi, votre action peut tout à fait avoir des effets thérapeutiques importants, je pense donc que la relation que vous avez avec le personnel pourrait vous aider dans le choix des enfants, car je suppose que vous n'avez pas le temps d'aller visiter chaque chambre chaque jour.

131 Jean-Claude FINES : Nous ne faisons pas des visites chaque jour, et ensuite ce n'est pas nous qui choisissons les enfants à visiter. 11 s'agit de la surveillante générale qui indique le service et les chambres. Actuellement, nous avons planifié jusqu'au mois de septembre. 
Donc, on sait absolument qui ira voir qui, et c'est l'infirmière en chef ou la surveillante qui décide qui va être visité, qui n'est pas trop fatigué par un traitement... À ce niveau, pas de problème.

Au niveau des infirmières, nous allons essayer de leur apprendre quelques tours de magie ou de clowns, de façon à ce qu'elles aient ces petits plus afin de faire en sorte que les enfants, lorsqu'ils sortent des points par exemple, aient l'esprit ailleurs.

Et inversement, nous essaierons pour les artistes, d'expliquer quels sont les problèmes que peut ressentir un enfant, de les former spécifiquement au milieu hospitalier, le tout sans aller trop loin, car il faut garder à l'idée que l'enfant reste normal.

Pascal FORCIOLI : Je voudrais simplement intervenir en complément de ce que vient de dire M. FINES. Je crois que là on montre bien sur cet exemple que des partenaires de l'hôpital interviennent dans la fonction soin. C'est vrai que l'infirmière dit que cela contribue à la thérapeutique, il faut que cela contribue à la thérapeutique. Cela fait partic malgré tout de votre mission, ce n'est pas uniquement divertir. C'est aussi introduire le monde civil, le monde ludique, mais cela fait partie de la thérapeutique et de l'approche thérapeutique.

135 A ce titre, la question que l'on doit se poser est celle de savoir si cette action doit être financée de manière externe à l'hôpital, ou si au contraire, l'hôpital doit intégrer dans ses frais de fonctionnement cet aspect de ce qui devrait être son activité. Personnellement, j'ai tendance à considérer en tant que directeur d'hôpital que je n'ai pas envie de faire la manche pour payer ce genre d'activité. Et je voudrais pouvoir le faire sur mon budget de fonctionnement. Et je trouve gênant quelque part d'être obligé d'apporter ce plus, comme l'embellissement d'un bureau, d'une zone... Je trouve qu'il est dommage que l'on soit obligé en permanence d'aller faire la manche auprès des laboratoires, des associations, des collectivités locales... parce que cela crée parfois des situations de dépendance dans des relations clients-fournisseurs. Et puis ce n'est pas sain que l'on soit obligé d'aller chercher ailleurs ce dont on a besoin en fait pour fonctionner. Et je pense également que le fait que cette reconnaissance n'existe pas, marginalise quand même quoi qu'on en dise, les partenariats qui peuvent exister. Il y a un partenariat qui existe, mais faute d'être reconnu, intégré, il reste marginal, il n'est pas répandu.

\section{Synthèse de Christian MOREAU}

136 Nous avons démarré l'après-midi sur la notion de réseau, et nous nous sommes posés des questions, et cela était essentiel, sur le partenariat. Je crois que cela a été le mot clé, le leitmotiv de chacun des intervenants. Et nous avons terminé par l'expression de l'un des partenaires dont on a parlé, et j'ai trouvé cela très bien. Quand on voit le thème de cette table-ronde qui était « La prise en compte des partenaires de l'hôpital dans l'œuvre de santé: innovations et expérimentations", on se pose chaque fois que l'on parle d'expérimentation des questions d'éthique, et je remercie Mme COLDBERC d'avoir posé le problème en termes d'éthique et de déontologie des intervenants dans le champ sanitaire. Je crois qu'elle est essentielle. Quand on expérimente, il faut toujours réfléchir à ce que l'on fait, au but essentiel de ce que l'on fait. Quand on parle de santé publique, il faut penser qu'il s'agit avant tout de la santé du public et que c'est aussi la nôtre demain en tant qu'usager du service hospitalier. 
137 Autre leitmotiv : le décloisonnement : hôpital / extrahôpital, champ sanitaire / champ social. Effectivement, si l'on construit des réseaux, c'est pour décloisonner. Nous avons ressenti un souci de professionnalisme dans chacune des interventions : c'est dire qu'il n'y a plus de place pour l'improvisation. Il faut absolument savoir où l'on va, et quelqu'un a dit, M. FINES, qu'il avait le souci de ne pas communiquer trop tôt. Je pense qu'il résume assez bien la chose : faisons d'abord, faisons savoir ensuite.

Merci de votre attention.

\section{AUTEUR}

\section{CHRISTIAN MOREAU}

Journaliste à «Objectifs soins» 